\title{
Three dimensional analysis of the retinal vasculature using immunofluorescent staining and confocal laser scanning microscopy
}

\author{
D M Foreman, S Bagley, J Moore, G W Ireland, D McLeod, M E Boulton
}

\begin{abstract}
Aim-To undertake a qualitative and quantitative analysis in three dimensions of the human retinal vasculature.

Method-Fixed and excised whole retinas were permeabilised and subjected to immunofluorescent staining for blood vessel components followed by confocal laser scanning microscopy. Single projection and stereoimages were constructed using computer software. $\mathrm{XZ}$ sections through the retina were constructed and the vasculature analysed using appropriate software.

Results-Immunofluorescent staining with no discontinuities was present in vessels of all sizes, the confocal images of the capillary network being free of out of focus blur at all depths. Quantitative analysis of $\mathrm{XZ}$ sections confirmed the qualitative impression of sharp delineation of the deep retinal capillary plexus, an absence of laminar arrangement of capillaries within the inner retina, and a truncated cone of capillaries around the foveal avascular zone (FAZ) wherein the superficial capillaries approached the FAZ more closely than those in the deeper retina.

Conclusion-Immunofluorescent staining of the retina and confocal laser scanning microscopy were shown to be useful in analysing accurate three dimensional reconstructions of the normal retinal vasculature without affecting overall tissue architecture.
\end{abstract}

(Br F Ophthalmol 1996; 80: 246-251)

Department of
Ophthalmology,
University of
Manchester
D M Foreman
J Moore
D McLeod
M E Boulton

School of Biological Sciences, University of Manchester

D M Foreman

$S$ Bagley

J Moore

$G$ W Ireland

M E Boulton

Correspondence to: Dr David Foreman University Department of Ophthalmology, Manchester Royal Eye Hospital, Oxford Road, Manchester M13 9WH.

Accepted for publication 9 November 1995
The microcirculation of the retina has been the subject of extensive investigation owing to its importance in retinal maintenance and its role in retinal disease. ${ }^{1-8}$ The spatial distribution and functional integrity of the retinal vessels can be determined in vivo using fluorescein angiography which permits the visualisation of arterioles, venules, and macular capillaries in two dimensions. However, even in the optimal conditions of an immobile globe and a uniformly highly pigmented retinal pigment epithelium (which blocks choroidal fluorescence and thus maximises contrast), fluorescein angiography does not demonstrate the deeper capillaries in the inner retinal plexus in the macula owing to light scatter within the retina. ${ }^{9}$

Laboratory methods of studying the three dimensional structure of the retinal circulation include digest, ${ }^{3}$ colloidal iron, ${ }^{12}$ and corrosion cast techniques. ${ }^{10-12}$ Such well established techniques are complex, often require freshly enucleated eyes, and depend on the liberation of the vasculature from its surrounding supporting tissue. We have devised a simple technique which permits a comprehensive examination of the spatial network of capillaries in the retina using immunofluorescent staining, confocal microscopy, and computer analysis. This report presents our findings in the normal human retina.

\section{Materials and methods}

PREPARATION OF TISSUE

Five human eyes were obtained from the Manchester Royal Eye Hospital eye bank following removal of the corneoscleral rim for grafting. Donor ages were $43,54,64,72$, and 92 years and eyes were received in the laboratory within 24 hours post mortem. The anterior segment of each eye was removed by a circumferential incision just posterior to the ora serrata. The vitreous and neuroretina were detached gently from the retinal pigment epithelium, cut free at the optic disc, and removed. The whole retina was then gently teased free from the majority of the vitreous and fixed overnight at $4^{\circ} \mathrm{C}$ in $10 \%$ neutral buffered formalin. Retinas were washed with phosphate buffered saline (PBS) and permeabilised by immersion in $0.2 \%$ Triton X-100 in PBS for 4 hours.

\section{IMMUNOFLUORESCENT STAINING}

The retinal vasculature was immunostained either for von Willebrand factor ( $v W f$ ) (factor VIII related antigen), a specific marker for endothelial cells, or for type IV collagen, a component of the blood vessel basement membranes. Non-specific binding was blocked by a 1 hour treatment with $2 \%$ normal goat serum in PBS containing $0.2 \%$ Triton X-100. All subsequent antibody and buffer solutions contained $0.2 \%$ Triton and $0.2 \%$ normal goat serum. Retinas were incubated overnight with a 1:50 dilution, in PBS, of either rabbit antihuman vWf antibody (Dako) or mouse anti-human collagen IV (Dako).

Retinas were then washed in PBS for 3 hours before addition of either FITC conjugated goat anti-rabbit IgG or FITC conjugated goat antimouse IgG (Sigma) (1:40 in PBS). After overnight incubation, the retinas were washed for 3 hours in PBS and flat mounted vitreous side up in Gelvatol. ${ }^{13}$ Fluorescent staining was 
viewed using either conventional fluorescence microscopy on an Zeiss photomicroscope III or by confocal microscopy (see below). Negative controls were prepared by omitting the $1^{\circ}$ antibody. Conventional fluorescence photographs (Tmax 400 film, Eastman Kodak) were taken of representative fields of microvasculature using the inbuilt camera.

\section{CONFOCAL MICROSCOPY}

Specimens stained with fluorescent probes were examined with an MRC 600 confocal laser scanning attachment (Biorad) mounted on a suitably modified photomicroscope III (Zeiss) using a BHS filter block: $4 \times$ (Olympus, numerical aperture $[\mathrm{NA}]=0 \cdot 13), \quad 10 \times$ (Olympus, $\mathrm{NA}=0.3$ ), $16 \times$ (Zeiss, $\mathrm{NA}=0.5$, working distance $[\mathrm{WD}]=0.15 \mathrm{~mm}$ ), and $40 \times$ (Zeiss, NA $=0.9$, WD $=0.3 \mathrm{~mm}$ ) multi-immersion objectives were used with a minimum pinhole size. A series of images $(768 \times 512$ pixels) were captured at different depths within the specimen typically separated by 3-10 $\mu \mathrm{m}$. Each stored image was a Kalman average of five captured images and the completed $\mathrm{Z}$ series was stored on optical discs using an optical drive (Panasonic 7030E).

Computer software enabled the information to be presented in several different ways for qualitative analysis.

\section{Single maximum projection image}

Using computer software a series of images, captured at regular depths (3-10 $\mu \mathrm{m}$ intervals) throughout the retina, was stacked and a projection image constructed.

\section{Stereopairs}

Laterally shifting each image in the stack of images by one pixel to the left and to the right created two separate maximum projections which appeared in black and white stereo if viewed with an appropriate stereo viewer.

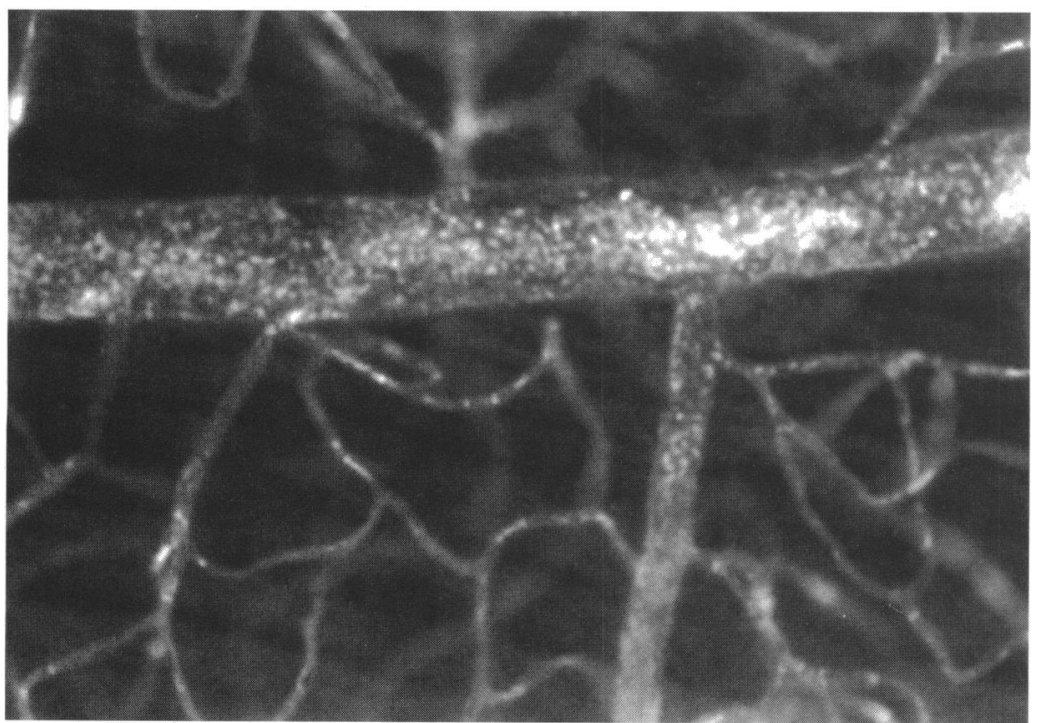

Figure 1 Conventional fluorescence photomicrograph of a region, $7 \mathrm{~mm}$ superior to the foveal avascular zone, of a retina from a 43-year-old person stained for von Willebrand factor. The superficial capillaries and larger vessel are in focus and sharply defined, whereas the deeper capillaries are blurred and out of focus. Magnification $\times 200$.
Stereo anaglyph

The same pair of images was colour coded and used to create a red green stereo anaglyph which was then viewed with appropriate red green spectacles.

\section{Computer reconstruction}

$\mathrm{XZ}$ sections in any plane and with any part of the data set were constructed. The data were transferred to a Silicon Graphics Indigo 2 workstation and converted (using software written by $M$ Lee and D Hancock, Department of Computer Science, University of Manchester) from pixels into cubic voxels. Using an $x$ ray network within AVS (Advanced Visualisation Systems Inc), which performs simple orthographic volume visualisation on three dimensional fields, the image was viewed in the $\mathrm{Z}$ direction. In some cases, to accentuate the outlines of the microvessels, an edge detector filter (Adobe Photoshop 2.5) was used.

Captured images were converted to TIFF format using the program $\mathrm{X}$-image (Foster Findlay) and output to a CI-5000 slide writer (Polaroid). RGB stereo anaglyphs were created by colouring and merging stereo pairs using Adobe Photoshop software and output in a similar way.

\section{QUANTITATIVE ANALYSIS OF THE VASCULAR} BED

$Z$ series (step size $3 \mu \mathrm{m}$ ) were collected at various points temporal to the foveal avascular zone (FAZ) from a retina stained for vWf. Each image of a $Z$ series was processed using Semper 6 plus (Synoptics, Cambridge, UK). Using a two point recursive filter (rf) the images were strongly smoothed. Each smoothed image was then subtracted from its parent image to produce a confocal image which had more of an even background pixel spread. The images were reconstituted back into a $\mathrm{Z}$ series which was then transferred to a Silicon Graphics Indigo 2 workstation. The data were converted from pixels into cubic voxels and transferred to the Kendall Square Research 1-64. After thresholding to produce binary images containing only positively stained voxels, the volume of the stained vessels was calculated and expressed as a percentage of the total volume of the $Z$ series. The width of the vascular network was determined by multiplying the number of images in the $\mathrm{Z}$ series containing positive staining for $\mathrm{vWf}$ (that is, containing vessels) by the step size between images. The reproducibility of the technique was demonstrated by collecting three $Z$ series from the same area of retina, $10 \mathrm{~mm}$ temporal to the FAZ. These were then processed for volume analysis and found to be almost identical (mean 7.9 (SEM 0.3)).

\section{Results}

In our illustrations and terminology, we have adopted the convention that the retina is orientated vitreous surface up, photoreceptors down, and that objects in the retina 


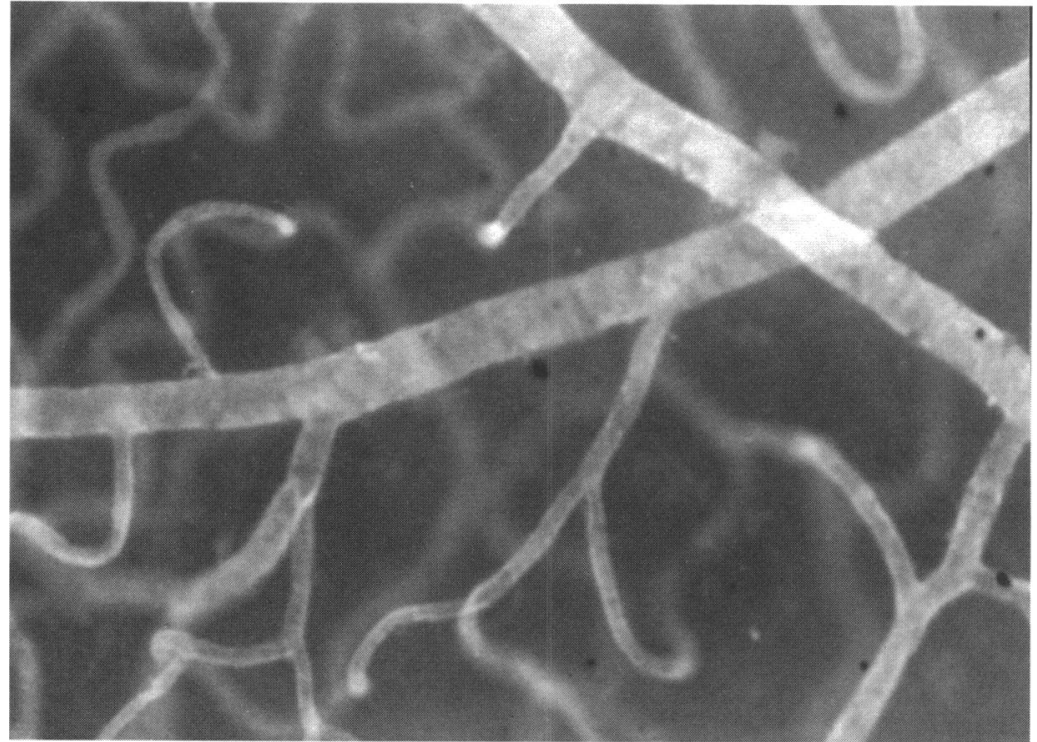

Figure 2 Conventional fluorescence photomicrograph of a region, $6 \mathrm{~mm}$ superior to the foveal avascular zone, of a retina from a 64-year-old stained for type IV collagen. The uniformly stained superficial capillaries are in focus. Magnification $\times 200$.

termed superficial are towards the vitreous surface whereas objects termed deep are more scleral.

\section{CONVENTIONAL MICROSCOPY}

When flat retinal preparations stained for $\mathrm{vWf}$ were viewed by epifluorescence microscopy the retinal capillaries appeared as a three dimensional network during refocusing; the spatial arrangement could be captured only imperfectly by conventional microscopy (Fig 1). The demonstration of retinal vessels was comprehensive and extended from large vessels to the
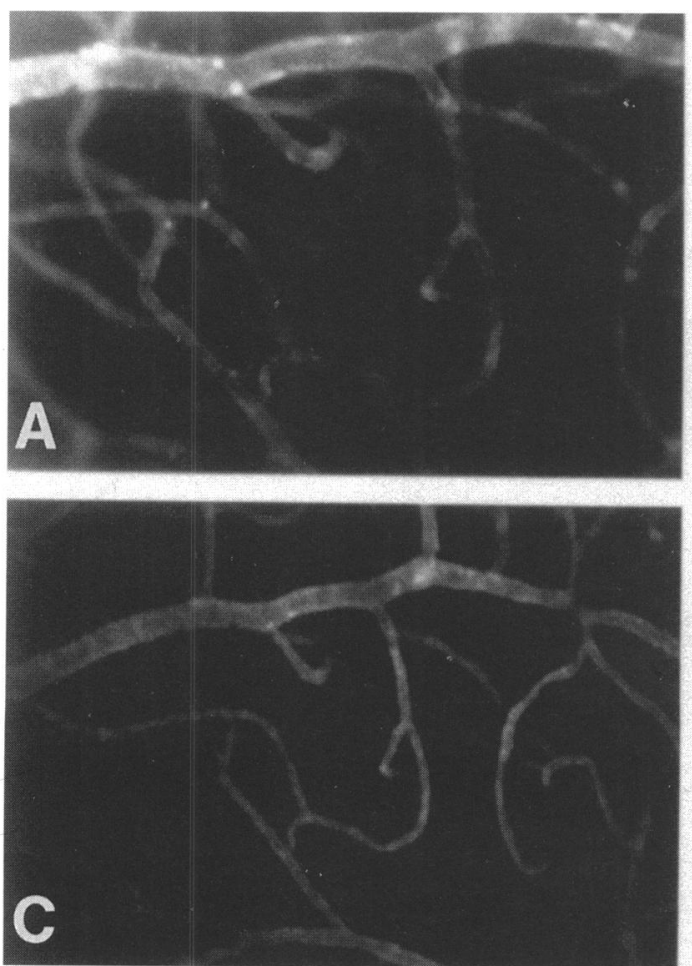

very finest of capillaries with no discontinuities. The staining intensity for $\mathrm{vWf}$ was variable among and within vessels. The granular pattern observed in vessels larger than capillaries appeared to be due to perinuclear staining of stored $\mathrm{vWf}$ within organelles known as Weibel-Palade bodies. Type IV collagen immunostaining gave a similar demonstration of the retinal microvasculature, but vessels were delineated with a smoother, regular, and more uniform outline than that obtained with vWf (Fig 2). Interestingly, the inner limiting lamina, the basement membrane forming the boundary between the retina and the vitreous, did not stain for type IV collagen. Controls, in which the primary antibody was omitted, showed no staining of vessels.

\section{CONFOCAL MICROSCOPY}

Using the confocal microscope, images of the capillary network at various depths within the retina could be obtained. Furthermore, these images were free from out of focus blur. To illustrate, Figure $3 \mathrm{~A}$ shows a conventional microscopic image of an area of retina from a 64-year-old human, stained for collagen IV, captured $8 \mathrm{~mm}$ temporal to the FAZ. Figure 3B shows a confocal image of the deepest network of fine capillaries in the same area of retina. This network appeared to have a discrete outer limit. A confocal image captured $60 \mu \mathrm{m}$ above that of Figure 3B shows the less dense network of superficial capillaries (Fig 3C). The single maximum projection image demonstrates the entire vasculature in focus in two dimensions throughout the field of view (Fig 3D).
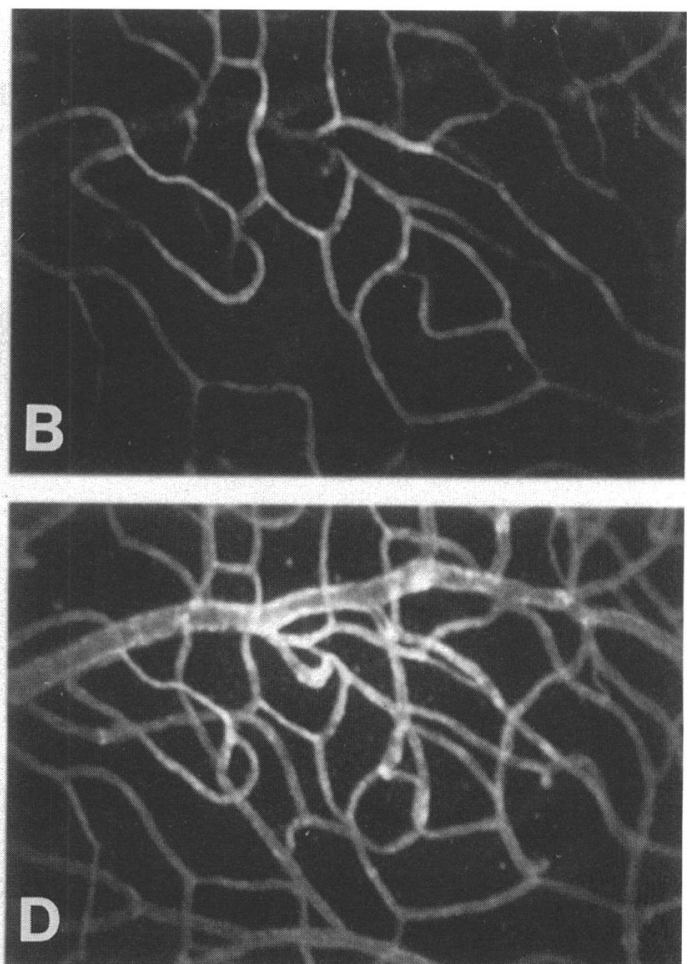

Figure 3 (A) Conventional fluorescence photomicrograph of region, $8 \mathrm{~mm}$ temporal to the foveal avascular zone, of a retina from a 64-year-old human stained for type IV collagen. (B) Confocal image of the same area of retina as (A) captured at the outer boundary of the capillary plexus. Sharp image in the confocal plane. (C) Confocal image captured $60 \mathrm{\mu m}$ above that shown in (B). Sharp image of a single superficial capillary segment. (D) Confocal projection image of the same region of retinal - that is, 20 images, separated by $3 \mu \mathrm{m}$, were captured throughout the retina to complete a $Z$ series and used to create the single maximum projection image. The entire network is in focus. Magnification $\times 120$. 


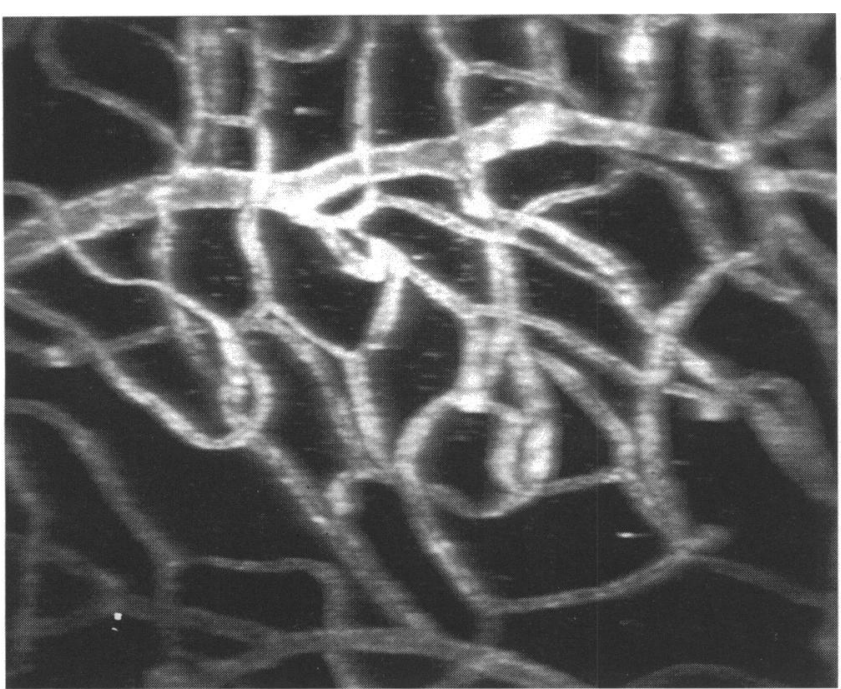

Fig $4 A$

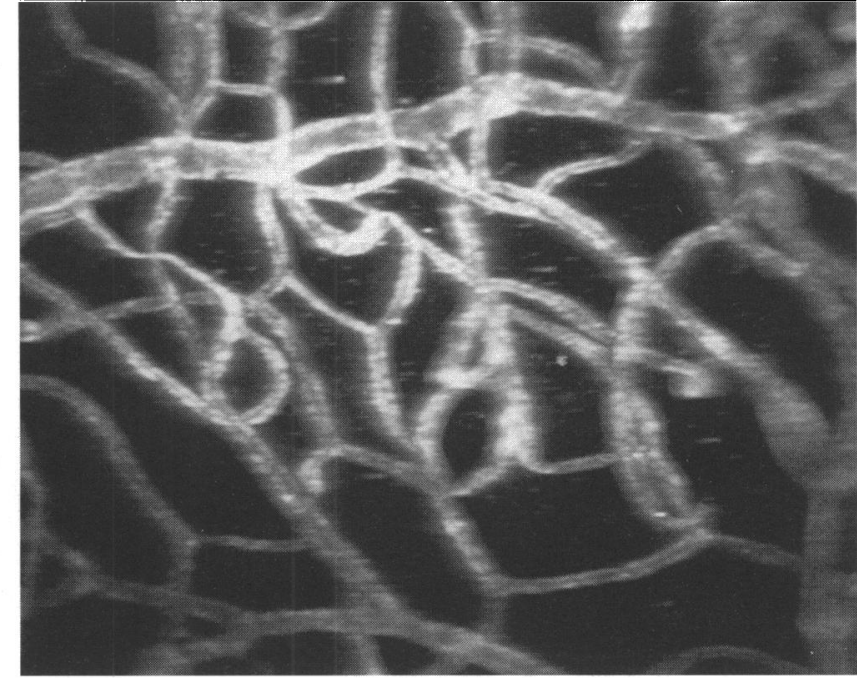

Fig $4 B$

Figure 4 (A) and (B). A stereo pair of the same maximum projection confocal image as in Figure $3 D$. Magnification $\times 150$.

The three dimensional images generated (Figs 4 and 5) confirmed the qualitative findings described above. Within the superficial network were the larger venules and the arterioles from which arose capillaries at oblique, often steep, angles. They then connected with the venular capillaries at all depths with little or no evidence of layering.

An XZ section of a confocal $\mathrm{Z}$ series taken $2.5 \mathrm{~mm}$ superior to the FAZ of a 92-year-old man clearly revealed the relations between the individual capillaries but provided no evidence of a laminar distribution of capillaries between the upper and lower limits (Fig 6).

$\mathrm{XZ}$ sections of the confocal stack of images collected at the fovea showed that the terminal loops of the capillaries, as they approached the FAZ, form a truncated cone in which the superficial capillaries approached the FAZ more closely than those in the deeper retina (Fig 7).

VASCULAR BED WIDTH AND VOLUME ANALYSIS After further processing of collected $Z$ series, the width and volume of the vascular network

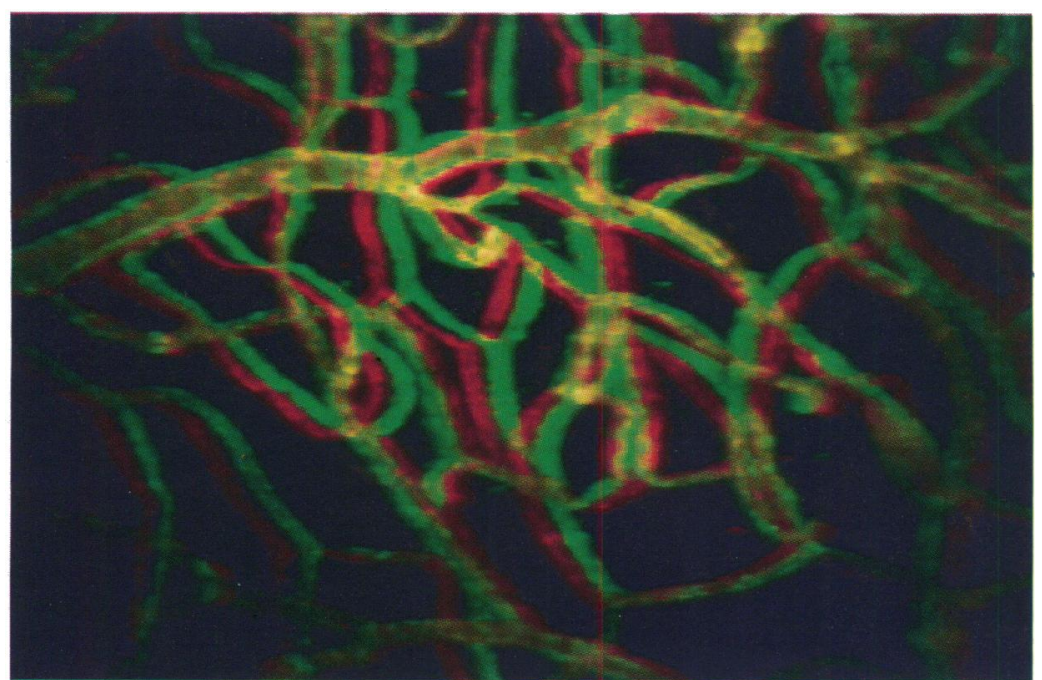

Figure 5 Confocal stereo anaglyph of the same maximum projection confocal image as in Figure 3D. Using red/green spectacles it is possible to observe the three dimensional structure of retinal vessels as visualised from the vitreous side of the retina and showing no laminar arrangements of capillaries. Magnification $\times 150$. at various points temporal to the FAZ were analysed (Table 1). The capillary network around the FAZ from a 72-year-old retina was typical of the five eyes and was approximately $97 \mu \mathrm{m}$ thick and the volume of positive staining for vWf (used as a measure of the volume of the capillary network) $17 \%$. These values were $94 \mu \mathrm{m}$ and $28 \% 2 \mathrm{~mm}$ from the FAZ, fell to $78 \mu \mathrm{m}$ and $13 \% 8 \mathrm{~mm}$ from the FAZ, and to $47 \mu \mathrm{m}$ and $4 \%$ in the peripheral retina, $15 \mathrm{~mm}$ from the FAZ.

\section{Discussion}

A number of techniques have previously been used to study the architecture of the blood vessel network in normal and diseased eyes. All have their limitations, particularly in providing an accurate three dimensional picture of the inner retinal network and in identifying subtle changes associated with diseases such as early diabetic retinopathy. In order to visualise the vessels in three dimensions, the circulation has been injected with (i) citrated red blood cells and gelatin, followed by benzidine peroxidase staining of the erythrocytes to delineate the vessels, ${ }^{2}$ or (ii) water to lyse the red cells followed by formal saline and colloidal iron (Indian ink) ${ }^{12}$ or (iii) neoprene latex (and latterly methyl methacrylate) producing a plastic vascular cast which, after pepsin/trypsin or $\mathrm{KOH}$ digestion of extravascular tissues, was studied using the dissecting microscope or scanning electron microscope. ${ }^{10-12}$ However, these techniques suffer from the difficulty of ensuring complete filling, and avoiding overdistension, of the

Table 1 Thickness and volume of the retinal vascular network of a retina from a 72-year-old at different distances temporal to the foveal avascular zone (FAZ)

\begin{tabular}{lll}
\hline $\begin{array}{l}\text { Distance from } \\
F A Z(\mathrm{~mm})\end{array}$ & $\begin{array}{l}\text { Vascular bed } \\
\text { thickness }(\mu \mathrm{m})\end{array}$ & $\begin{array}{l}\text { Volume } \\
(\%)\end{array}$ \\
\hline 2 & $94(7)$ & $27 \cdot 7(7 \cdot 8)$ \\
8 & $78(6)$ & $12 \cdot 9(5 \cdot 1)$ \\
15 & $47(3)$ & $4 \cdot 0(1 \cdot 6)$ \\
\hline
\end{tabular}

$\star$ At each point analysis was performed on three areas (each $0.27 \mathrm{~mm}^{2}$ ). Values are mean (SEM). 


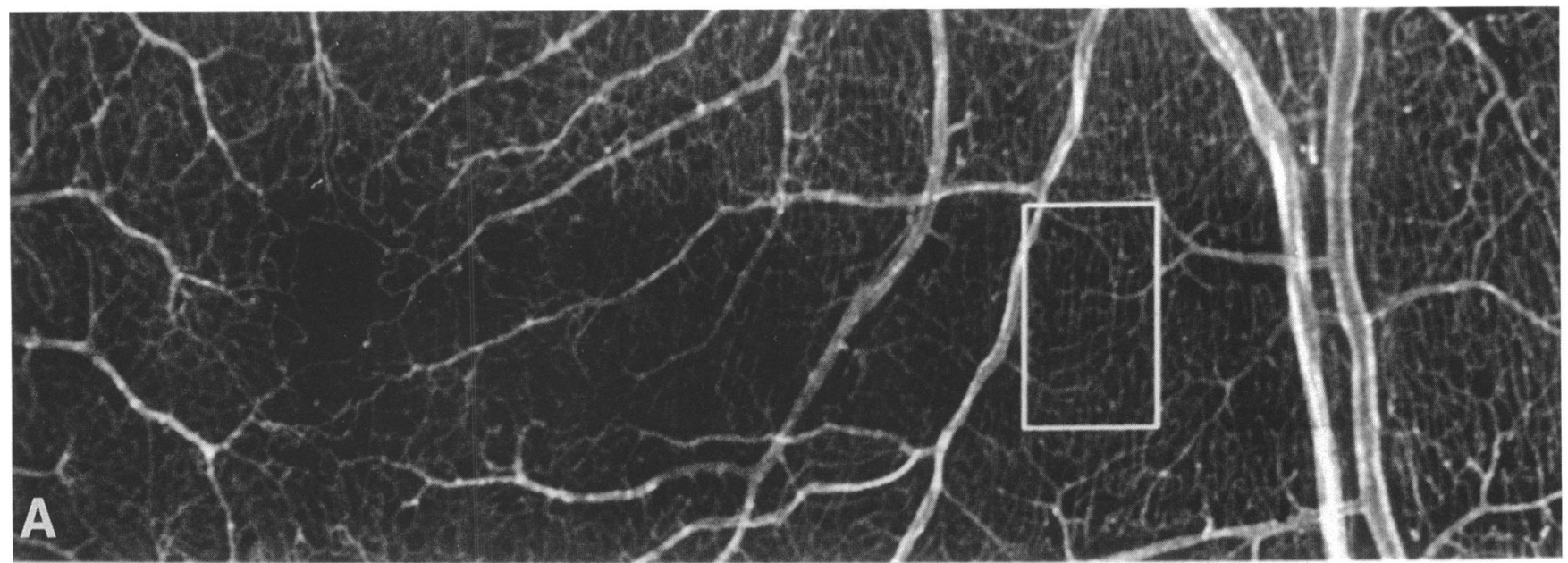

(1)

Figure 6 (A) Montage of images taken with the confocal microscope from the foveal avascular zone to the superior temporal arcade of a 92 year old male stained for type IV collagen. Images were obtained using $a \times 4$ lens, with a wide pinhole allowing a large depth of field, and clearly demonstrate the comprehensive staining of all vessels of all sizes. Magnification $\times 47$. (B) Confocal projection image of capillaries contained in the boxed area in $(A)$. Using the $\times 16$ lens, 35 images, step size $3 \mu \mathrm{m}$, were collected to form a $Z$ series and used to create a maximum projection image. Magnification $\times 188$. (C) $X Z$ section of a portion (boxed area) of the $Z$ series collected in (B), demonstrating the depth and spacing of the individual capillaries but indicates there is no laminar distribution of capillaries in this section of retina.
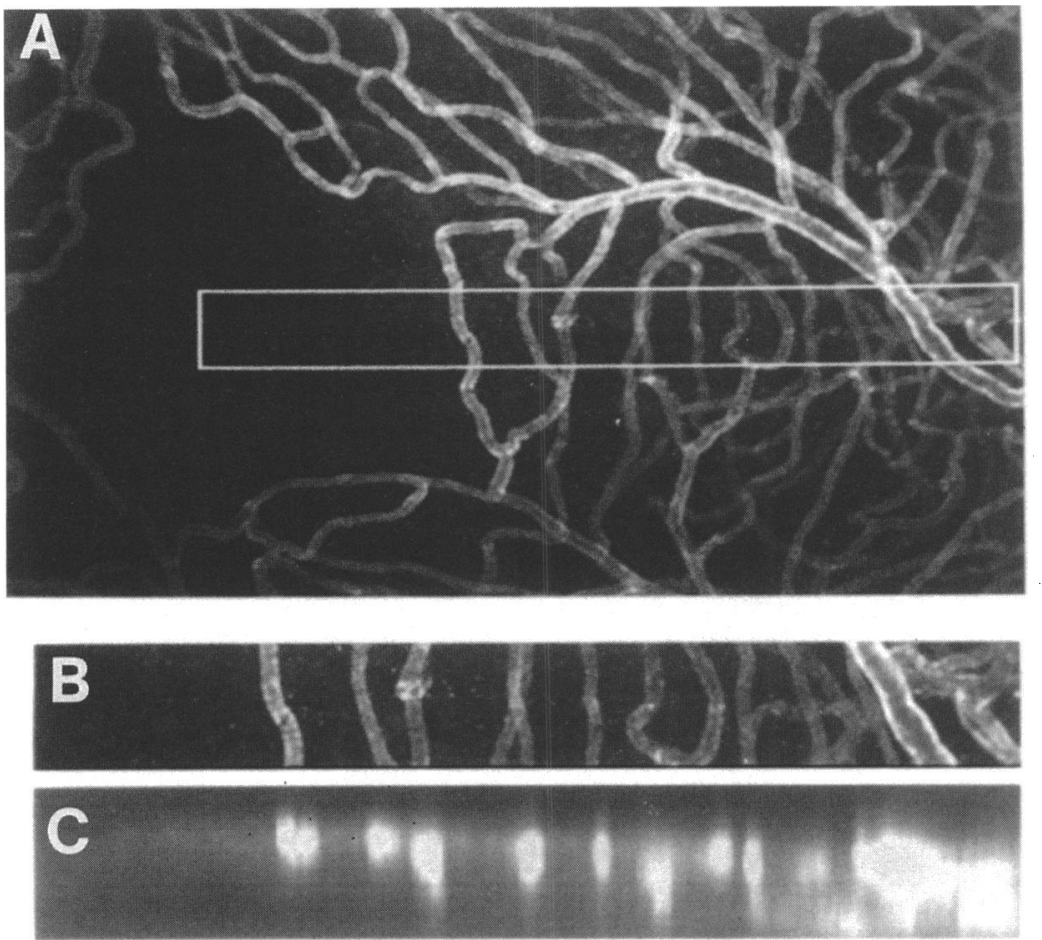

Figure $7 \quad X Z$ section through the terminal loops surrounding the foveal avascular zone of a retina from a 72 year old. Eighteen confocal images, separated by $5 \cdot 2 \mu \mathrm{m}$, were taken to construct a $Z$ series data set and projected as a maximum projection image $(A)$, part of which (hatched box) was extracted (B) and rotated through $90^{\circ}(C)$. The XZ image $(C)$ showed that superficial capillaries approached the fovea more closely than those in the deeper retina. Magnification $\times 100$. vessels as well as generally requiring freshly enucleated eyes.

The paucity of collagen in the retina and the relative resistance of the vascular bed to trypsin digestion have enabled the retinal circulation to be studied at the light microscopic level wherein the endothelial and pericyte components of the undigested microvasculature can generally be distinguished. ${ }^{3}$ The digest can even be resuspended to allow three dimensional study of the capillary bed, ${ }^{4}$ but such digests may fail to maintain their in vivo spatial orientation owing to the absence of supporting cells and matrix components. To overcome many of these drawbacks several optional but complicated approaches have been reported recently including those by (i) Krauss ${ }^{5}$ who injected hydrogen peroxide into the vitreous body of whole human eyes; the conversion of hydrogen peroxide to oxygen by erythrocyte catalase resulted in the accumulation of gas within the retinal vessel lumens and, following complex tissue preparation, the gas filled vessels were viewed by dark field microscopy, (ii) Snodderly and co-workers ${ }^{7}$ who laboriously mapped the capillaries of undigested retinal whole mounts using drawing tubes mounted on a microscope and traced the continuity of the vessels by changing the focus with vessel segments assigned to their respective depth planes, and (iii) Lutty and McLeod ${ }^{6}$ who used an enzyme histochemical based technique, exploiting the high adenosine $5^{\prime}$ diphosphatase 
activity in retinal endothelial cells, to visualise retinal blood vessels.

The simple staining technique described in this study, together with the use of confocal microscopy, permit the three dimensional reconstruction of the retinal vasculature with greater accuracy than that obtained by other techniques. The confocal microscope and its computer software has the advantage that it provides an accurate three dimensional reconstruction of features (for example, the vasculature) within a whole tissue without affecting overall tissue architecture. Furthermore, once collected the three dimensional data sets can be further analysed for surface area, volume, length, width, and orientation of the capillaries either in each plane or in the whole $\mathrm{Z}$ series. In addition, this technique can be undertaken on fresh or fixed retina and does not require whole eyes, the technique being applicable to small segments of retina. Furthermore, once the vasculature has been mapped accurately it is possible to embed the retina for wax/resin histology. Cut sections for histology of areas of interest - for example, for neurovascular correlation, can then be taken.

Using a confocal laser scanning microscope we have collected images of the capillary network at various depths within the normal retina and have generated a three dimensional reconstruction of the retinal vasculature. The overall pattern and density of the vascular network varied with both retinal region and retinal depth. Our observations using the confocal microscope demonstrated a remarkably clear outer delineation of the deep network of capillaries but no evidence of a laminar distribution of capillaries between these limits as was originally proposed by Michaelson, ${ }^{2}$ confirmed by Snodderly et $a l^{7}$ in subhuman primates, but refuted by Toussaint et al. ${ }^{4}$ The large vessels (venules and arterioles) were randomly distributed at different depths throughout the superficial regions only - that is, they were absent from the deep plexus. Only capillaries were observed immediately adjacent to the FAZ and, in agreement with the findings of Toussaint et $a{ }^{4}{ }^{4}$ they were shown to surround an avascular truncated cone.

While our technique is ideally suited for mapping the capillary network of normal and diseased retinas, by utilising various markers in combination (for example, against endothelial cells, pericytes, and type IV collagen) it may be possible to further understand the mechanisms of capillary closure and dropout, events known to be among the earliest changes in diabetes mellitus. ${ }^{14}$ The technique described here is not restricted to three dimensional analysis of the retinal vascular network but can also be used to identify and quantify the spatial location of other components in the retina (for example, non-vascular cells, growth factors, extracellular matrix components). Our technique offers the ability to (i) comprehensively study the three dimensional structure of the human retinal plexus, (ii) determine the spatial and topographical changes in aging and disease (for example, diabetic retinopathy), and (iii) correlate changes in perfusion and vascular permeability with alterations in specific vascular cells, extravascular cells, and extracellular matrix components.

The authors would like to thank the Guide Dogs for the Blind Association, the Manchester Royal Eye Hospital Endowment Fund, the British Diabetic Association, the Medical Research Council, and the Royal Society for their support. We are grateful to Professor M W J Ferguson for use of the Silicon grateful to Professor $M$ W J Ferguson for use of the Silicon
Graphics workstation and to $M$ Lee and D Hancock for their Graphics
software.

1 Ashton N. Injection of the retinal vascular system in the enucleated eye in diabetic retinopathy. $\mathrm{Br} 7 \mathrm{Ophthalmol}$ 1950; 34: 38-41.

2 Michaelson IC. Retinal circulation in man and animals. Springfield, IL: Charles C Thomas, 1954.

3 Kuwabara T, Cogan DG. Studies of retinal vascular patterns: normal vascular patterns. Arch Ophthalmol 1960 patterns: nor

4 Toussaint D, Kuwabara T, Cogan DG. Retinal vascular patterns: II. Human retinal vessels studied in three dimensions. Arch Ophthalmol 1961; 65: 575-81.

5 Krauss $R$. New technique to demonstrate the network of blood capillaries of the human retina in their three-dimensional arrangement. Graefes Arch Clin Exp Ophthalmol 1990; 228: 187-90.

6 Lutty GA, McLeod DS. A new technique for visualization of the human retinal vasculature. Arch Ophthalmol 1992, 110: $267-76$

7 Snodderly DM, Weinhaus RS, Choi JC. Neural-vascular relationships in central retina of macaque monkeys (Macaca fascicularis). $\mathcal{F}$ Neurosci 1992; 12: 1169-93.

8 Stitt AW, Anderson HR, Gardiner TA, Archer DB. Diabetic retinopathy: quantitative variation in capillary basement membrane thickening in arterial or venous environments. Br f Ophthalmol 1994; 78: 133-7.

9 Weinhaus RS, Burke JM, Delor FC, Snodderly DM. Comparison of fluorescein angiography with microvascular anatomy of Macaque retinas. Exp Eye Res 1995; vascular an

10 Wybard KC. Anastomoses between the retinal and ciliary circulation. Br F Ophthalmol 1956; 40: 65-81.

11 Shimizu K, Ujiie K. Structure of vascular vessels. Tokyo, New York: Igake Shoin, 1978.

12 Risco J, Nopanitay W. Ocular microcirculation. Scanning electron microscope study. Invest Ophthalmol Vis Sci 1980; 19: 5-12.

13 Rodriquez J, Deinhardt F. Preparation of a semi-permanant mounting medium for fluorescent antibody studies. Mounting medium for

14 Ashton N. Pathogenesis of diabetic retinopathy. In: Little HL, ed. In: Diabetic retinopathy. 1983: 85-106. 